\section{Measuring the performance of more circular complex product supply chains}

\author{
Ellen Bracquené ${ }^{1}$, Wim Dewulf ${ }^{1}$, Joost R. Duflou ${ }^{1}$ \\ 1. KU Leuven, Departement of Mechanical Engineering, Celestijnenlaan 300A Box 2422, 3001 Leuven, Belgium
}

Contact info: ellen.bracquene@kuleuven.be

Keywords: circular economy; product circularity indicator; material efficiency; environmental trade-off; LCA

\title{
Abstract
}

This paper researches the possibility to measure the performance of more circular complex product supply chains. Although a number of circularity indicators have already been proposed in literature, none was found to properly describe the product system taking into account the 'tightness' of the material cycles and the relationship with other product systems such as the use or supply of recycled material. Therefore, a new Product Circularity Indictor (PCl) is developed in this paper. The ability of the $\mathrm{PCl}$ to overcome the main limitations identified is demonstrated in a comparative study with the existing Material Circularity Indicator $(\mathrm{MCl})$. In addition, the new indicator is applied and tested in a case study for Washing Machines (WM). The case study results show that the proposed $\mathrm{PCl}$ is a useful indicator to quantify the effectiveness of different circular economy (CE) strategies. A shift to CE presents the challenge of recirculating material flows in a manner that can promote eco-effectiveness. Therefore the potential trade-off between increasing circularity and minimising the environmental burden of the WM is investigated using Life Cycle Assessment (LCA) to quantify the potential environmental impact of the product system.

\section{Introduction}

The ambition to 'Live well within the limits of our planet' has guided policy-makers around the world to define specific goals and action plans [1]-[3]. The limits of our planet are described in a comprehensive manner by Rockström [4]. The planetary boundaries are defined for a wide range of environmental processes that are affected by anthropogenic perturbations. However, the amount of resources that can be extracted without the risk of destabilizing the Earth System (ES) has not been defined. Natural resources, although extracted from the environment, are a man-made concept and limitations from social and environmental impacts are likely to result in economic scarcity well before physical depletion occurs [5]-[7]. This explains the difficulty of assessing resource depletion as an environmental impact category [8], [9] despite the growing concern for future limitation in terms of resource inputs and waste sinks [10].

It is widely recognized that the current linear supply chain based on a discard oriented society is not sustainable and that there is a need for transition towards an economy that will decouple economic progress from resources depletion [11]-[13]. The Circular Economy (CE) concept summarizes different 
approaches that can contribute to this overarching goal [14]-[16]. A shift to circular economy presents the challenge of recirculating material flows [17] in a manner that can promote eco-effectiveness [18]. Different strategies exist for the restoration of material flows such as repair, preserving the product as a whole, refurbishment, preserving the use of components or, as a last resort, recycling the material. The strategies are complementary to each other because they act at a different stage of the product cycle. This paper researches the possibility to measure the performance of more circular complex product supply chains. In this context, circularity is defined as the ability to conserve both the quantity and the quality of the material. The quality conservation can partially be described through the tightness of the material circle which encourages to maintain products (and components) at their highest level of value for as long as possible [19].

Several authors have investigated the definition and use of circularity measures [17], [20]-[28]. Circularity can be assessed at different levels ranging between micro or product-level, meso or (inter)company-level and macro or (inter)regional-level [29]. Macro-level indicators, generally based on Material Flow Analysis (MFA), have been more widely applied and researched compared to micro-level indicators [10], [17], [30][32]. However, micro-level indicators are necessary to capture the effect of potential interventions at product level where many CE strategies are put into practice. The three most commonly cited micro-level indicators are the Material Circularity Indicator ( $\mathrm{MCl}$ ), proposed by the Ellen MacArthur Foundation (EMF) and Granta Design (GD) [22], the Circular Economy Index (CEI) proposed by Di Maio and Rem [33] and the Reuse Potential Indicator (RPI) proposed by Park and Chertow [34]. Linder et al. conclude the $\mathrm{MCl}$ is one of the most promising and ambitious attempts yet to develop a product-level circularity metric [17]. In their state of the art analysis of CE measures, Elia et al. also found that, at micro level, the proposed $\mathrm{MCl}$ indicator managed to incorporate most of the desired CE requirements [35]. Garza-Reyes et al. also considered the $\mathrm{MCl}$ to be the most complete assessment framework for micro-level circularity available in literature [23]. Different authors have selected the $\mathrm{MCl}$ to measure the circularity at micro-level in their analysis of the trade-off between material circularity and environmental efficiency [24], [36].

In this paper, the main limitations of the existing Material Circularity Indicator ( $\mathrm{MCl})$ are discussed and a new Product Circularity Indicator (PCI) is developed. The ability of the $\mathrm{PCl}$ to overcome the identified limitations is investigated in a comparative study with the $\mathrm{MCl}$. In addition, the $\mathrm{PCl}$ is applied and tested in a case study for Washing Machines (WM). Finally, the potential trade-off between increasing circularity and minimising the environmental burden is investigated using Life Cycle Assessment (LCA).

\section{Method for circularity assessment at product level}

The objective of the Material Circularity Indicator ( $\mathrm{MCl}$ ) developed by EMF and GD is to measure the extent to which the linear flow has been minimized and restorative flow maximized' [22]. A summary of the equations used in the $\mathrm{MCl}$ mathematical model are given in Table 1 and a detailed description of their 


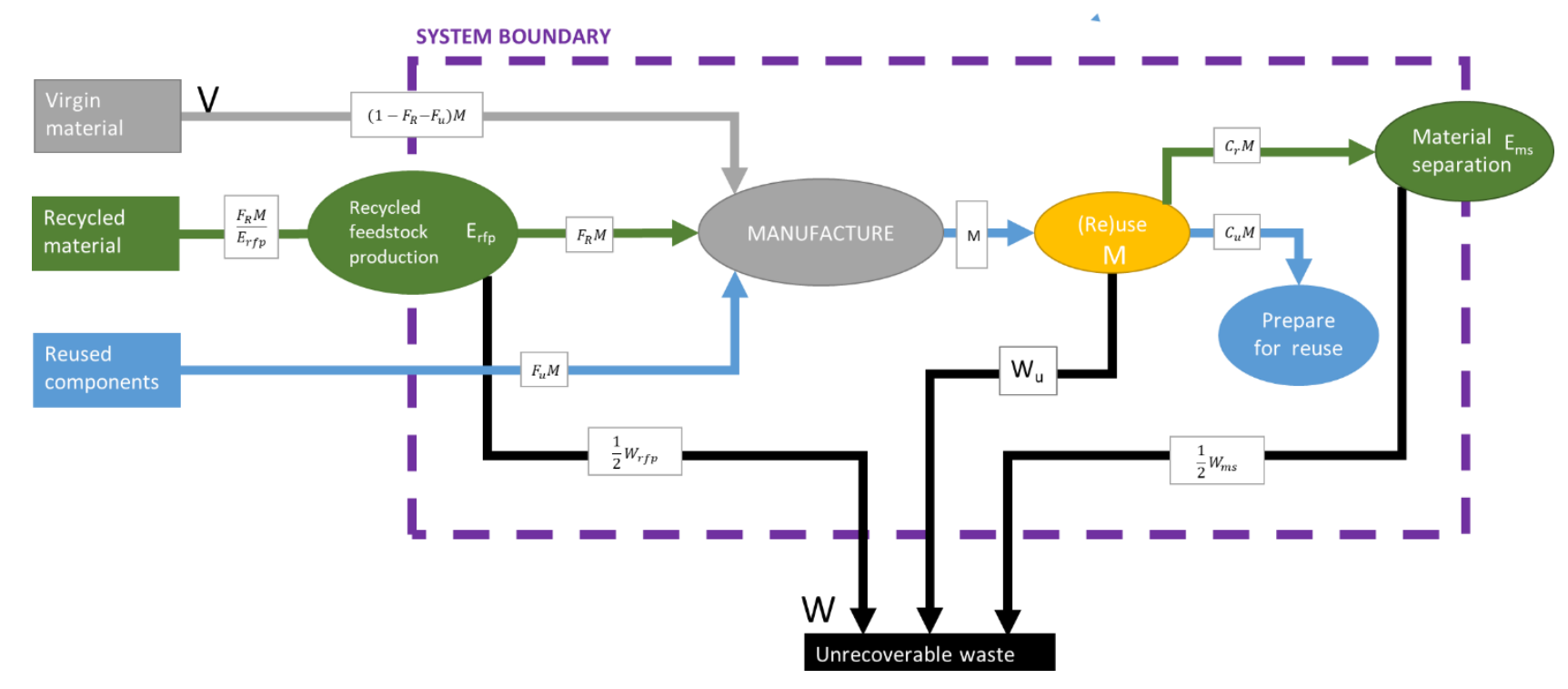

Figure 1: System boundary of the Material Circularity Indicator (MCl)

3 The product has a total mass $(M)$ and is partly manufactured from virgin feedstock $(V)$. A function $(F)$ is derived depending on the utility $(\mathrm{X})$ of the product in such a way that the $\mathrm{MCl}$ increases with improved utility. The total mass $W$ of unrecoverable waste that is attributed to the product system includes the uncollected waste after use $\left(W_{u}\right)$, waste generated during material recovery $\left(W_{m s}\right)$ and recycled feedstock production $\left(W_{r f p}\right)$. However, the authors only include half of the waste related to material recovery and recycling motivated by the 50/50 allocation rule [22]. According to the 50/50 allocation rule, the burden of shared processes are equally distributed between the previous and/or subsequent lifecycle of the product system studied. In the $\mathrm{MCl}$, the production of recycled material and the recovery of material at end-of-life (EoL) are considered shared processes.

The $\mathrm{MCl}$ aims to quantify the fraction of material flows that are circular or non-linear compared to a linear system. For a product with mass $M$, in case of a fully linear system, a mass $M$ of material flows in the system and another mass $M$ flows out at end-of-life. This would result in a denominator of $2 M$. However, due to the 50/50 allocation, it should be corrected for the amount of waste generated by the recycled feedstock production upstream allocated to the product system $\left(\frac{W_{r f p}}{2}\right)$. In addition, part of the waste generated by the material recovery at EoL is allocated to the subsequent product system using the recycled material $\left(\frac{W_{m s}}{2}\right)$. The $\mathrm{MCl}$ calculation method is therefore summarized by the following equations for the Linear Flow Index (LFI) and the Material Circularity Indicator (MCI):

$$
L F I=\frac{V+W_{u}+\frac{W_{r f p}}{2}+\frac{W_{m s}}{2}}{2 M+\frac{W_{r f p}}{2}-\frac{W_{m s}}{2}}
$$

$$
M C I=1-L F I . F(X)
$$

Although the $\mathrm{MCl}$ allows for reused components $\left(F_{u}\right)$ to enter the value chain, this flow does not displace new manufactured components. In the $\mathrm{MCl}$ model, only one manufacturing stage is defined which includes 
1 all production activities simultaneously (material production, component production and assembly). As a 2 consequence, the reused components are assumed to displace virgin material. In addition, the $\mathrm{MCl}$ 3 assumes that the flow of reused components and recycled material are fully circular, even though for some 4 flows only partial circularity can be accounted for within the considered product system. In order to be 5 fully circular, both the generation and the use of restorative flows must be demonstrated.

6 A first consequence of these assumptions and modelling choices is that the $\mathrm{MCl}$ is unable to account for 7 the 'tightness' of the material cycles (reuse vs. recycling) which can potentially have significant implication 8 for the effectiveness of the material cycling [17]. Secondly, the $\mathrm{MCl}$ completely ignores where the reused 9 components and recycled materials are sourced from and where the recovered components and materials will end up. Disregarding the relationship with other product systems, that absorb or generate recycled feedstock, can only be motivated if the recovered material is reused within the system boundary. In order to adequately describe a real-life open-loop product system, the exchange of components and recycled feedstock with other product systems has to be taken into account because as long as the loop is not fully closed it should not be accounted as such in the circularity metric.

In addition, the $\mathrm{MCl}$ does not take into account the effect of downcycling which can happen when the material degrades due to changes in inherent properties. In this case the material can no longer be used in the same or similar application. If downcycling is not incorporated in the circularity indicator, it is not able to account for the quality preservation of recovered and recycled materials.

Another limitation of the $\mathrm{MCl}$ is that only the recycled feedstock production is included while the other manufacturing stages, such as virgin feedstock production, are excluded in the main part of the methodology without any clear motivation. A first observation is that it would be more consistent to either include all or none of the manufacturing stages. In addition, even if only part of the manufacturing steps are included, the same cut-off should apply to both virgin and recycled material.

Finally, the fraction of recycled material content $\left(F_{r c}\right)$ and fraction of reused components $\left(F_{u}\right)$ are both defined at product or component level and are therefore not completely independent in the $\mathrm{MCl}$ model $\left(F_{r c}+F_{u} \leq 1\right)$. In reality feedstock is produced from a mix of virgin and recycled material and it would therefore be more practical to define the recycled content $\left(F_{r}\right)$ at material rather than at component or product level.

In this paper a novel method for circularity assessment at product level (PCI) is introduced to overcome the main limitations identified for the existing indicator $(\mathrm{MCl})$. The different manufacturing steps are considered and the associated material losses are accounted for as waste or recycled material. The inclusion of separate manufacturing steps allows for the different restorative flows to re-enter the production chain at the appropriate stage. The components harvested for reuse are assumed to avoid the production of new components while the recovered and recycled materials are assumed to reduce the need for virgin material. The feedstock production starts with the material processing step that includes both virgin and recycled material as input, such as ingot production for metals. Potential losses during the assembly stage are not included in the current $\mathrm{PCl}$ model. 
The material separation and recycled feedstock production are fully part of our product system which allows for clear boundary between product systems. However, in order to ensure mass balance, an exchange with a stock of 'recycled material' is included. If the product system produces more recycled material than it takes up, the surplus material will leave the system and will be added to the recycled material stock. If, on the other hand, the product does not recover sufficient recycled material after the use phase, recycled feedstock from the stock will be used as recycled content for the feedstock production. The same reasoning can be applied to components. However, most components are product specific and the exchange with other systems might not be practically feasible, expect for standardized components.

Figure 2 illustrates the model used to develop the $\mathrm{PCl}$ as described in this section of the paper. The equations to calculate the necessary material flows are derived in the next subchapters and summarized in Table 1.

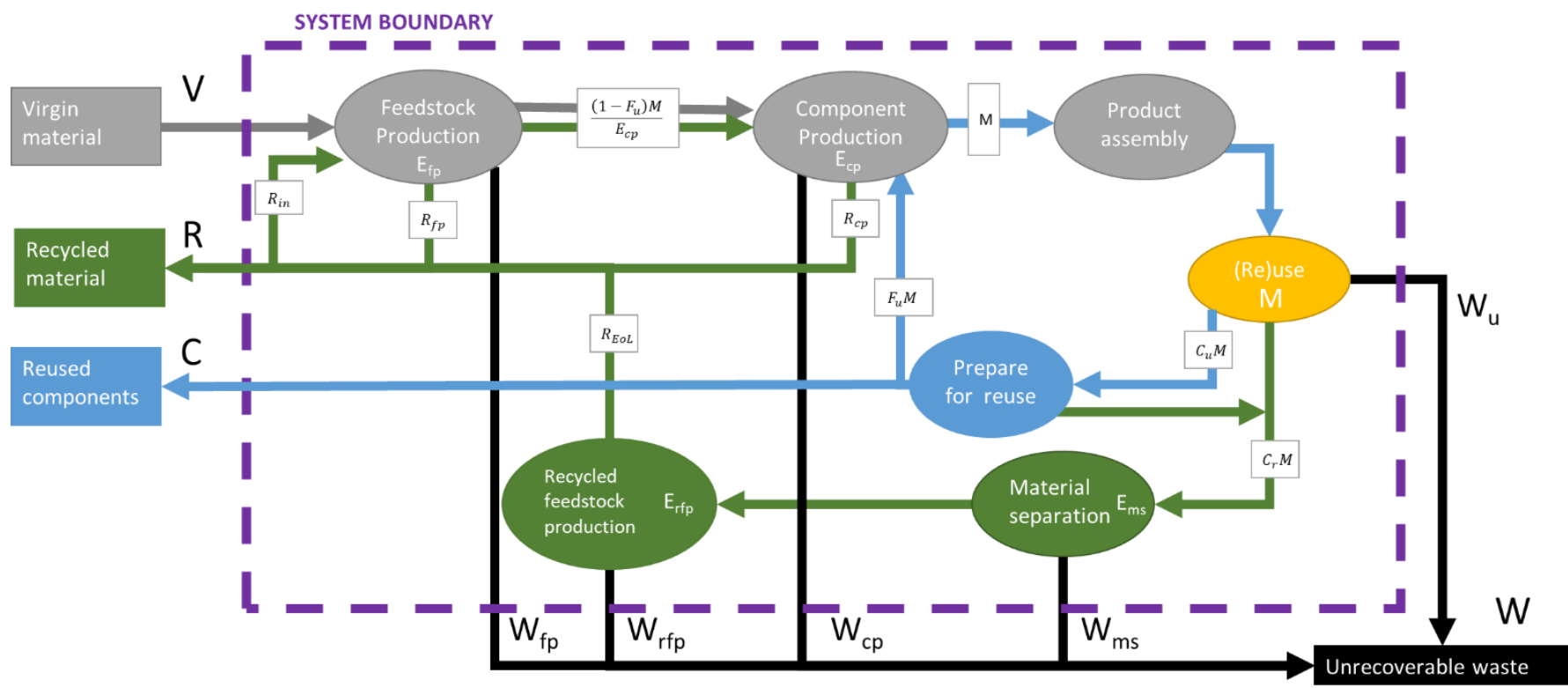

\subsection{Virgin material (V)}

The amount of required virgin material is derived from the known mass of the final product $(M)$. First, the fraction of reused components $\left(F_{u}\right)$ is deducted. Secondly, the production losses during component production and feedstock production are taken in account. $E_{c p}$ and $E_{f p}$ are the efficiency of the component and feedstock production. The manufacturing efficiencies determine the amount of material required upstream to cope with the subsequent losses down the supply chain. Finally, the amount of recycled content $\left(F_{r}\right)$ of the produced feedstock is deducted to calculate the amount of virgin material $(V)$ :

$$
V=\frac{\left(1-F_{u}\right) M}{E_{c p} \cdot E_{f p}}\left(1-F_{r}\right)
$$


$2 \quad W$ is the total amount of unrecoverable waste leaving the product system. The $\mathrm{PCl}$ model includes both manufacturing waste and post-use waste. Manufacturing waste includes waste from feedstock production $\left(W_{f p}\right)$ and waste from component production $\left(W_{c p}\right)$. However not all material loss during production is waste. $C_{f p}$ and $C_{c p}$ are the fractions of material losses that are recovered as useful recycled material. The manufacturing waste generated is calculated with the following equations taking into account the efficiency of the feedstock production $\left(E_{f p}\right)$ and of the component production $\left(E_{c p}\right)$ :

$$
\begin{gathered}
W_{f p}=\frac{\left(1-F_{u}\right) M}{E_{f p} E_{c p}}\left(1-E_{f P}\right)\left(1-C_{f p}\right) \\
W_{c p}=\frac{\left(1-F_{u}\right) M}{E_{c p}}\left(1-E_{c p}\right)\left(1-C_{c p}\right)
\end{gathered}
$$

The post-use waste includes the material sent to energy recovery or landfill at end-of-use $\left(W_{u}\right)$, waste generated during material separation $\left(\mathrm{W}_{\mathrm{ms}}\right)$ and waste generated during recycled feedstock production $\left(W_{\text {rfp }}\right)$. These waste streams can be calculated as follows:

$$
\begin{gathered}
W_{u}=M\left(1-C_{u}-C_{r}\right) \\
W_{m s}=M\left(1-E_{m s}\right) C_{r} \\
W_{r f p}=M E_{m s} C_{r}\left(1-E_{r f p}\right)
\end{gathered}
$$

$C_{u}$ represents the fraction of collected end-of-use products available for component reuse. Even though a product is collected for reuse, it is most likely not feasible to reuse all components. $C_{r}$ represents the fraction that is collected for recycling. The recycling consists of two distinct steps: material separation at end-of-life for resource recovery and further material processing to produce usable recycled feedstock. Efficiency factor $E_{m s}$ is the efficiency of the material separation and $E_{r f p}$ is the efficiency of the recycling process used to produce the recycled feedstock.

The total unrecoverable waste $\mathrm{W}$ can be calculated as follows:

$$
W=W_{f p}+W_{c p}+W_{u}+W_{m s}+W_{r f p}
$$

\section{3. $\quad$ Recycled material (R)}

In many cases the amount of recycled material generated by a product system does not match the amount of recycled material used in the manufacturing stage of the same system. Furthermore, the recovered material can often not be used for the same purpose due to quality losses with cascade recycling or downcycling as consequence. In most product lifecycles, there is either a recycled feedstock shortage or surplus. In the first case, recycled feedstock needs to be sourced from outside the product system. In the latter case the generated feedstock should be used outside the product system under investigation. The amount of recycled feedstock exchanged with the outer system $(R)$ depends on the amount of recycled material used as input $\left(R_{i n}\right)$, the amount of scrap generated during feedstock production $\left(R_{f p}\right)$ and component production $\left(R_{c p}\right)$, and the amount of end-of-life recycled material recovered $\left(R_{E o L}\right)$ : 


$$
R_{\text {in }}=F_{r} \frac{\left(1-F_{u}\right) M}{E_{f p} E_{c p}}
$$

$$
R_{f p}=\left(1-E_{f p}\right) C_{f p} \frac{\left(1-F_{u}\right) M}{E_{f p} E_{c p}}
$$

$$
R_{c p}=\left(1-E_{c p}\right) C_{c p} \frac{\left(1-F_{u}\right) M}{E_{c p}}
$$

$$
R_{E o L}=E_{r f p} E_{m s} C_{r} M
$$

$$
R_{\text {out }}=R_{f p}+R_{c p}+R_{E o L}
$$

$$
R=R_{\text {in }}-R_{\text {out }}
$$

\section{4. $\quad$ Reused components (C)}

Products can be collected for part harvesting to enable remanufacturing or repair. If the number of parts recovered exactly matches the number of parts used, there is no exchange across the system boundary. In other cases, the amount of material flowing through the system boundary for component reuse is calculated as follows:

$$
C=M\left(F_{u}-C_{u}\right)
$$

When collected parts can no longer be (re)used, they are assumed to be recycled.

\subsection{Utility factor $(\mathrm{X})$}

The utility factor aims to take into account how durable products are manufactured on the one hand and how intensively they are used on the other hand. The first part is mostly depending on the design and manufacturing stage. In other words, the "use potential" of a products depends on the manufacturer, but the final "used potential" depends on the user.

The reliability requirements for products are set by engineers in the manufacturing industry. These requirements determine the probabilistic need of satisfying specific product performance parameters across the product life cycle. The design life of a product $\left(L_{d}\right)$ is the period of time during which that product system is expected by its designers to perform intended functions within its specified design parameters and operational environment [37]. The design life is usually derived from the expected product life by the customers in their viewpoint and time scales such as years. After the expected design life is estimated based on market research, the design life in engineering terms or functional usage duty cycles $\left(F U D C_{d}\right)$ can be calculated by assuming a specific use intensity $\left(I_{d}\right)$ as design target. The product utility $X$ is defined as the ratio of the available or used $F U D C$ versus the expected $F U D C_{d}$ based on average product design requirements:

$$
X=\left(\frac{L}{L_{d}}\right)\left(\frac{I}{I_{d}}\right)=\frac{F U D C}{F U D C_{d}}=\frac{\text { Available or used functional units }}{\text { Expected functional units }}
$$


The denominator equals the number of functional units the product is designed to last for based on market average for a specific product group $\left(F U D C_{d}\right)$. The numerator represents the actual available or used functional unit depending on the perspective of the assessment. The manufacturer can increase the number of available functional units by designing a product for improved durability compared to market average $\left(F U D C>F U D C_{d}\right)$. Due to the difficulty to measure actual reliability of products put on the market, the available functional units can be based on the actual offering of the manufacturers which is the warranty period. The manufacturer will maximize the product reliability within this timeframe to minimize the warranty cost [38]. The consumer can increase the number of actual used functional units by increasing the use intensity (e.g. product sharing). The actual used functional units by the customers can be derived from consumer studies.

\subsection{Linear flow index (LFI)}

The Linear Flow Index $(L F I)$ is the fraction of material flowing through the system boundary in a linear fashion compared to the fully linear systems. The amount of material flowing in and out a fully linear system $\left(F_{r}=F_{u}=C_{u}=0\right)$, is computed as follows:

$$
V_{\text {linear }}=W_{\text {linear }}=\frac{M}{E_{c p} \cdot E_{f p}}
$$

The $L F I$ can then be computed as follows:

$$
L F I=\frac{V+W+\frac{1}{2}|R|+\frac{1}{2}|C|}{V_{\text {linear }}+W_{\text {linear }}}
$$

The recycled material and reused components, that are exchanged with other product systems, are in between a linear and circular flow. They do not count as linear flow because of their potential to be reused nor as fully circular because they depend on other product systems for either the generation or the use of the recycled material and reused components. If downcycling is not acknowledged, it does not stimulate the production of high quality secondary material and this could lead to accelerated degradation of the recycled material pool [39], [40]. Product systems generating recycled material or reused components should be rewarded for providing high quality material. On the other hand product systems using recycled or reused material should be rewarded for using low grade material. Although downcycling should not be encouraged, low grade application can broaden the possibilities for recycled feedstock when it is not possible to avoid quality degradation. If a quality factor $(Q)$ can be quantified that represents to what extent the inherent properties of the material are lost, the following equation could be used:

$$
L F I=\frac{V+W+Q_{\text {in }} R_{\text {in }}-Q_{\text {out }} R_{\text {out }}}{V_{\text {linear }}+W_{\text {linear }}}
$$

$R_{\text {in }}$ and $R_{\text {out }}$ can be calculated using equations 8-12. $Q_{\text {in }}$ and $Q_{\text {out }}$ are the quality factor of the material entering and leaving the product system respectively. The quality factor should be defined between $[0,1]$ with $Q=1$ representing a quality undistinguishable from virgin material. 
2 The $\mathrm{PCl}$ is calculated by considering the LFI and the utility $\mathrm{X}$ of the product in the following equation:

$$
P C I=1-\frac{L F I}{X}
$$

4 For products with a low utility $(X<1)$, the overall $\mathrm{PCl}$ computed with equation 19 can be a negative value.

5 For this reason the rule is added that if the $\mathrm{PCl}$ calculation turns negative, the $\mathrm{PCl}$ score is set equal to zero.

$6 \quad$ 2.8. Multi-material products

7 The $\mathrm{PCl}$ can be applied to a multi-material product using a mass-based weighting methodology:

8

$$
P C I_{\text {total }}=\frac{\sum_{i} M_{i} \times P C I_{i}}{\sum_{i} M_{i}}
$$

9 Equation 20 could also be used to calculate the individual $\mathrm{PCl}$ of each component. Lonca et al. have 10 demonstrated that such a disaggregation at component level would lead to minor deviations in the final 11 results at product level [24]. 
2 Table 1 provides an overview of the equations used in current proposed $\mathrm{PCl}$ calculation method. For easy comparison and to highlight the differences, the equations of the existing $\mathrm{MCl}$ method are included using a uniform symbol notation. The main differences between the $\mathrm{PCl}$ and $\mathrm{MCl}$ can be summarized as follows:

- The recycled content $\left(F_{r}\right)$ is defined at material level in the $\mathrm{PCl}$, while, in the $\mathrm{MCl}$, it is defined at product level $\left(F_{r c}\right)$.

- Material losses during feedstock and component production are considered in the $\mathrm{PCl}$. As a consequence, direct component reuse has more benefits compared to material recycling. This is a significant difference with the $\mathrm{MCl}$ method that only takes recycling efficiency into account.

- In the $\mathrm{PCl}$, material recovery and material recycling are considered to be fully part of the product system.

- Material flow exchanges with the outer system boundaries ( $R$ and $C$ ) are not accounted as fully circular in the $\mathrm{PCl}$ calculation method.

Table 1: Overview of equations used in $\mathrm{PCl}$ and $\mathrm{MCl}$ calculation models

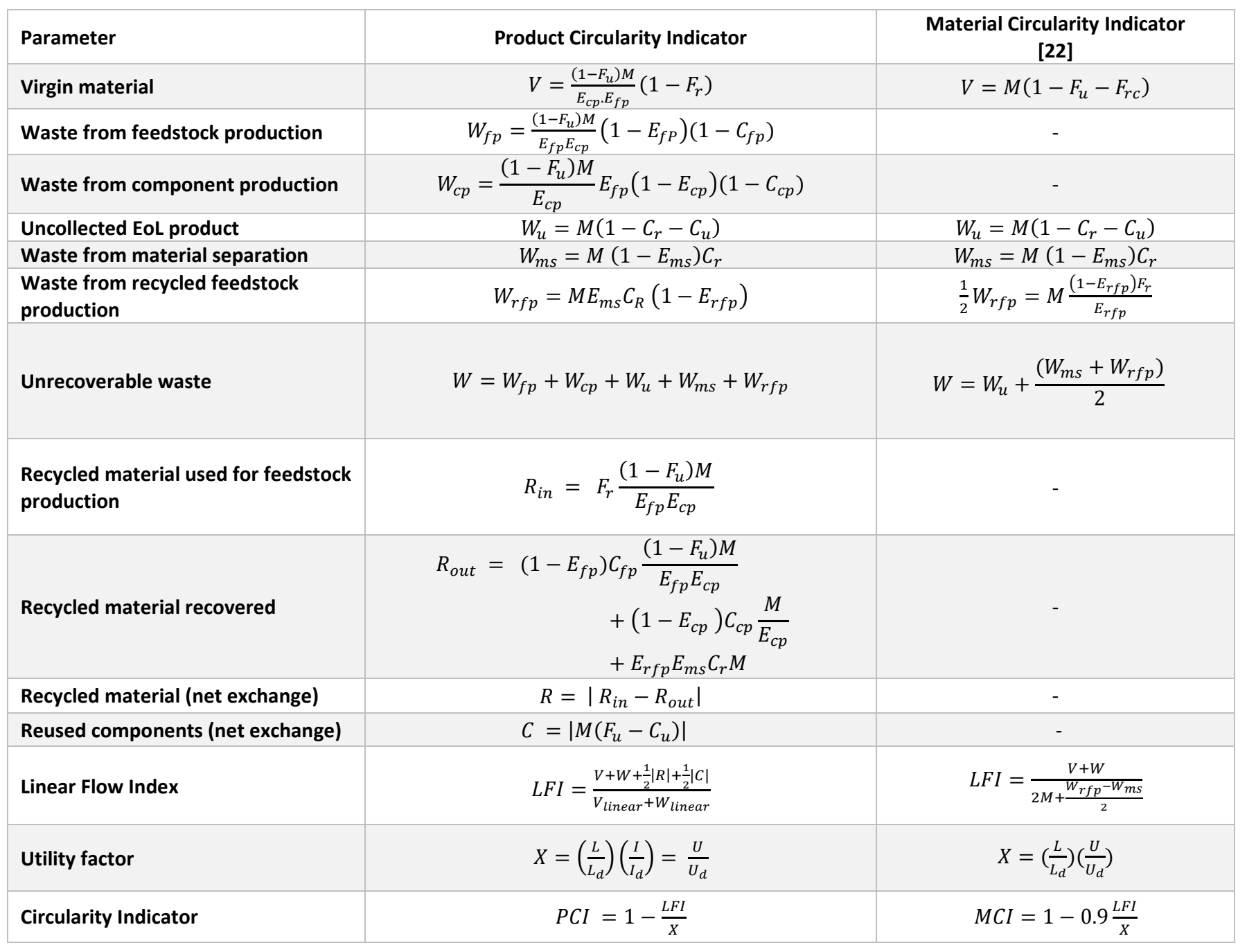


1 A sensitivity analysis is performed on a simple theoretical case to demonstrate the ability of the new $\mathrm{PCl}$ 2 to overcome the identified limitation of the existing $\mathrm{MCl}$. We assume a single material product with a 3 fraction of components $\left(F_{u}\right)$ that can be reused. The material feedstock is partly produced from recycled 4 material $\left(F_{r}\right)$. For the $\mathrm{MCl}$ calculations, we have to compute the recycled content at product level $5 \quad\left(F_{r c}\right)$ with the following formula:

$$
F_{r c}=\left(1-F_{u}\right) * F_{r}
$$

7 We further assume that all products are collected $\left(C_{r}=1-C_{u}\right)$ and a closed loop for the components $8\left(C_{u}=F_{u}\right)$. The efficiency of all the processes is assumed to be to 0.85 .

9 Figure $3(\mathrm{a})$ and Figure $3(\mathrm{~b})$ show the sensitivity of both $\mathrm{PCl}$ and $\mathrm{MCl}$ with a change of product reuse $\left(F_{u}\right)$ and recycled content of the material $\left(F_{r}\right)$. The results show that the $\mathrm{PCl}$ is much more sensitive to $F_{u}$ thus increasing the ability to reflect the tightness of the material cycle. In addition, the PCI behaves differently for the same increase in recycled content depending on the exchange with other product systems for the provision or absorption of recycled material. As long as the demand for recycled content is more than supplied $\left(R_{\text {in }}>R_{\text {out }}\right)$, an increase of $F_{r}$ will result in a higher increase of $\mathrm{PCl}$. Once $R_{\text {out }}=R_{\text {in }}$ the slope
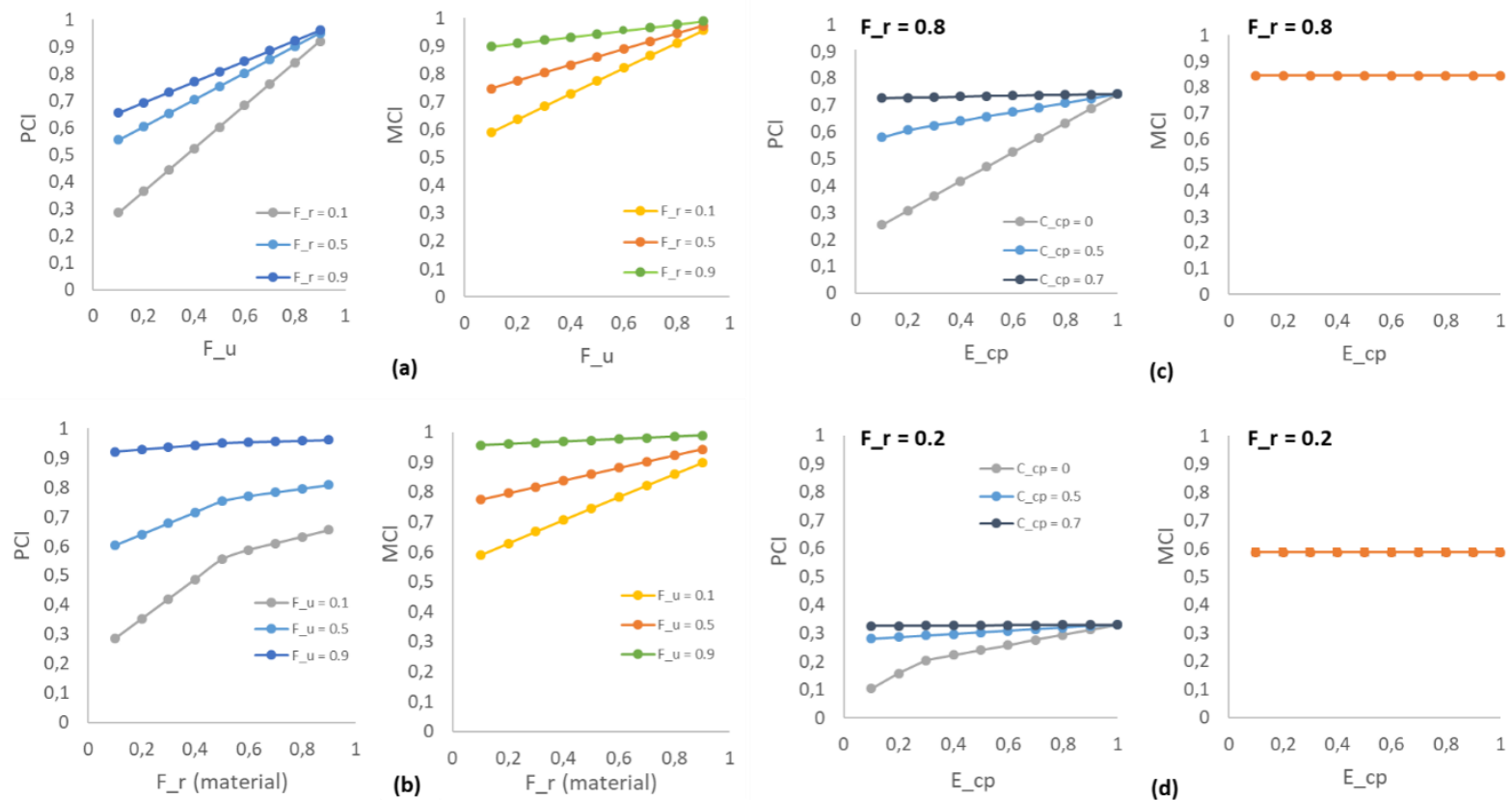
Figure 3: Comparison between new $\mathrm{PCl}$ and existing $\mathrm{MCl}$ for a change in (a) fraction of reused components $\left(\boldsymbol{F}_{u}\right)$, (b) recycled content of material $\left(F_{r}\right)$, (c) component production efficiency $\left(E_{c p}\right)$ for high recycled content (d) component production efficiency $\left(E_{c p}\right)$ for low recycled content

Further analysis is done to demonstrate the added value of incorporating a more detailed material flow during the production stage. For this analysis, the components of the single material product are assumed not to be reused $\left(F_{u}=C_{u}=0\right)$. In addition, the product is assumed to be collected for recycling at endof-use $\left(C_{r}=1\right)$. The efficiencies of the waste treatment steps are assumed to be $0.85\left(E_{m s}=E_{p r f}=\right.$ $0.85)$. For simplicity, only the efficiency of the component production $\left(E_{c p}\right)$ is varied while the efficiency 
1 of the feedstock production is not taken into account $\left(E_{f p}=1\right)$. Two different cases are considered. The 2 first assumes the product is made from feedstock with a high recycled content $\left(F_{r}=0.8\right)$ and the second 3 assumes low recycled content $\left(F_{r}=0.2\right)$. For each case, the fraction of recycled material recovered during 4 manufacturing $\left(C_{c p}\right)$ is varied from 0 to 0.7 .

5 Figure 3 (c) and Figure 3 (d) show the sensitivity of the $\mathrm{PCl}$ with a change in manufacturing efficiency $\left(E_{c p}\right)$ 6 for high and low recycled content. The influence of $E_{c p}$ is more important for products made from 7 materials with a high recycled content. On the other hand, the influence is minimized as more material 8 loss during production is recovered for recycling $\left(C_{c p}\right)$. As expected, the $\mathrm{MCl}$ is not affected by this 9 parameter and remains the same independently of $E_{c p}$. Even with a low recycled content $\left(F_{r}=0.2\right)$, the $10 \mathrm{MCl}$ is relatively high. This is due to the surplus of recovered 'recyclable' material at end-of-life $\left(C_{r}=1\right)$ 11 that is assumed to be fully circular in the $\mathrm{MCl}$ model. In future, as collection rates are improved, a more 12 detailed micro-level circularity calculation method, such as the $\mathrm{PCl}$, will become increasingly relevant to 13 allow differentiation between product systems. 
2 In this section, the developed $\mathrm{PCl}$ is applied to a real-life case study. The purpose is to demonstrate the practicability of the indicator and, in addition, to show the ability to investigate a number of improvement strategies based on CE thinking. WMs have a longer technological cycles which makes them a relevant candidate for CE strategies such as reuse and refurbishment. In previous research, WMs are often taken as an example to investigate eco-design measures such as durability [41], [42], repairability [43]-[45] or eco-efficiency [46]-[48].

\subsection{Data collection}

\section{Bill of Material}

A summary of all the materials used in a WM and their respective weight is given in Figure 4. This summary is based on the Bill of Materials (BOM) received from a manufacturer. The total weight of the WM is $69.51 \mathrm{~kg}$ excluding packaging. The received dataset contains detailed information for the contribution of each plastics type, but not for the type of steel used or for the exact composition of the electronic parts which include precious metals. Literature data are used to fill these data gaps. Ashby et al. reported that $59.9 \%(\mathrm{w} / \mathrm{w})$ of the steel parts from a WM were manufactured from mild steel, $16.2 \%$ from High Strength Low Alloy (HSLA) steel, 14\% from stainless steel (SS) and 9.9\% from cast iron [49]. The printed wiring board (PWB) composition is estimated based on data from Oguchi et al. [50]. In this study a number of PWBs from different product types are analysed and the concentration of different elements is determined. Based on this concentration and the known weight of the PWB in the WM, the amount of the different (precious) metals is calculated.

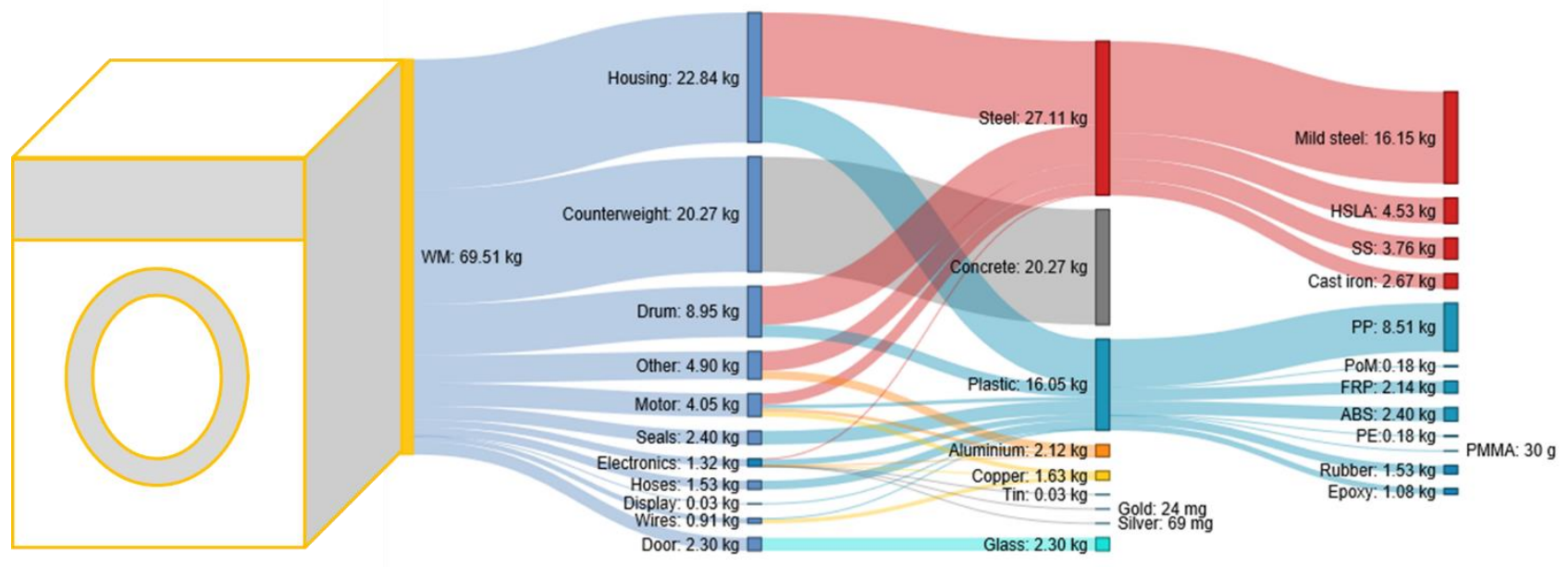

Figure 4: Composition of the case study washing machine

\section{Recycled content of feedstock}

The recycled content is defined as a material property. Average industry data is retrieved from literature for each material type. Ashby et al have determined the recycled content per steel type [49]. The recycled content of cast iron is around $69 \%$ while the HSLA steel is assumed not to contain recycled steel. Mild steel and stainless steel have an average recycled content of $42 \%$ and $38 \%$ respectively. For concrete, Cullen et al. estimate a recycled content of only $2 \%$ [20]. Kaweki et al. have done a probabilistic material flow 
analysis (MFA) for several plastic commodities in Europe [51]. For Polypropylene (PP), which is the most commonly used plastic in WMs, the recycled content is estimated to be around $12 \%$ assuming all recovered PP is reused as feedstock. Considering the numerous challenges to overcome, from separating the different polymer fractions during waste treatment to ensuring sufficient quality of the recycled feedstock, this is an optimistic assumption for the WM. Collecting meaningful and comprehensive recycling statistics has proven very difficult for the aluminium industry because there are numerous remelting and refining plants worldwide which can switch from scrap-based to primary-based production depending on market prices and product requirements at any time [52]. Nevertheless, the dynamic material flow model presented by Bertram et al. estimates that the global recycled content of aluminium is $52.6 \%$. Based on the dynamic model of global copper stocks and flows presented by Glöser et al., overall recycled content is calculated and equals $35.31 \%$ [53]. Eventhough the use of recycled cullets in glass production can amount to $50 \%$ of the material input, the glass used in electrical appliances is a specialty commodity representing only $2 \%$ of the glass sector [54]. Due to its small size and specific application, no recycled content is assumed for the glass used in the WM.

\section{Manufacturing losses}

The manufacturing of products includes feedstock production and component production. For the metal materials, the feedstock production starts with the ingot production and delivers half-fabricates. Table 2 summarize the manufacturing efficiencies for feedstock production $\left(E_{f p}\right)$ and component production $\left(E_{c p}\right)$ based on the available literature. In addition, the fraction of material loss recovered for recycling is also retrieved from literature when relevant for feedstock production $\left(C_{f p}\right)$ and for component production

$21\left(C_{c p}\right)$.

Table 2: Overview of average manufacturing efficiencies based on literature values for global production

\begin{tabular}{|l|c|c|c|c|c|}
\hline Material type & $\boldsymbol{E}_{\boldsymbol{f p}}$ & $\boldsymbol{C}_{\boldsymbol{f} \boldsymbol{p}}$ & $\boldsymbol{E}_{\boldsymbol{c} \boldsymbol{p}}$ & $\boldsymbol{C}_{\boldsymbol{c} \boldsymbol{p}}$ & Reference \\
\hline Steel & $73.95 \%$ & $43.35 \%$ & $87 \%$ & $99 \%$ & {$[55]$} \\
\hline Concrete & $99 \%$ & - & $99 \%$ & - & - \\
\hline Plastic & $100 \%$ & - & $99.5 \%$ & - & {$[51],[56]$} \\
\hline Aluminium & $70.5 \%$ & $95 \%$ & $78 \%$ & $96.8 \%$ & {$[57]$} \\
\hline Copper & $95.5 \%$ & $0 \%$ & $75 \%$ & $100 \%$ & {$[53]$} \\
\hline Glass & $97 \%$ & - & $97 \%$ & - & - \\
\hline
\end{tabular}

Rüdenauer et al. have compared the use information for WM based on data received from the manufacturer [48] . Back in 1991, a manufacturer has designed his products to last for 3500 wash cycles (14 years x 250 washes/year). Ten years later, in 2001, the design life decreased slightly to 3135 wash cycles (15 years $\times 209$ washes/year). Nowadays, 2500 wash cycles is a commonly assumed design life $\left(U_{d}\right)$ in industry (10 years $\times 250$ washes/year) [21], [37], [41], [58]. These data suggest that the design life has decreased with $28 \%$ over the last three decades.

31 Chen et al. analysed real life warranty data from a Chinese manufacturer, revealing that 3\% of the sold WM failed within the first 3 years and 50\% failed within 13.6 years [38]. Unfortunately, the use intensity of the devices was not monitored. Accelerated life tests (ALT) represent a methodology able to investigate 
1 product reliability performance in a shorter time compared to the conventional standard testing methods, 2 both in the design and in the production phase. Based on such experiments, a reliability of $99.2 \%$ for 500

3 cycles and $89.78 \%$ for 1250 cycles at normal user condition was derived by Borgia et al. [21], [58] which 4 indicates that the product has a high reliability (>99\%) in the first 2 years.

5 In most regions, household appliances are sold with a warranty period. Even though in some countries a 6 legally binding minimum is applicable, some manufacturers offer an extended warranty period for the 7 product or for a number of components. The technical call rate (TCR) for electronic products, defined as 8 share of products that fail during the warranty period, is often targeted to remain below $3 \%$ by internal 9 company policies [43]. This means that products are often designed for a reliability of $97 \%$ within the given warranty period. For WM, 3 additional years are common practice [42]. Together with the legal minimum, this usually results in a total of 5 years which covers $50 \%$ of the design life.

12 The actual consumer behaviour with respect to WM has been analysed at the University of Bonn. This study found that the average number of washing cycle per year in Europe is decreasing due to smaller household size and higher load per wash cycle [59]. The results varied significantly per household size with 2.2 cycles per week for a single person household and 6.8 for a household with at least five persons [59]. Boyano et al. estimate an average lifespan of 12.5 years for WMs for an normal use (220 wash cycle per year) [60].

Table 3 provides an overview of the derived utility factors. For the baseline, a default utility $(X)$ of 1 is assumed.

Table 3: Overview of derived utility factors

\begin{tabular}{|l|c|c|c|c|}
\hline Perspecticve & $\begin{array}{c}\text { Lifetime } \\
\text { (Years) }\end{array}$ & $\begin{array}{c}\text { Intensity } \\
\text { (Washes/year) }\end{array}$ & $\begin{array}{c}\boldsymbol{X} \\
(-)\end{array}$ & 0.2 \\
\hline Manufacturer - default warranty & 2 & 250 & 0.5 & - \\
\hline Manufacturer - extended warranty & 5 & 250 & 1 & {$[42]$} \\
\hline Baseline assumption & 10 & 250 & 1.1 & {$[41]$} \\
\hline Consumer - average & 12.5 & 220 & {$[60]$} \\
\hline
\end{tabular}

\section{Refurbishment and component reuse}

23 Consumer repair and reuse is included in the previous section on utility and is therefore not considered here. This section is about the reuse of components or refurbishment performed by a professional reuse centre or by a manufacturing company. The latter would require a take-back scheme or a product as a service business model.

In Europe, the number of Product Service Systems (PSS) is increasing and Bluemovement is an example of such an initiative in the Netherlands ${ }^{1}$. It is founded by a WM manufacturer and allows customers to subscribe to the use of a WM rather than owning a WM. The customer can choose between a refurbished WM at less than 10 Euro/month and a new WM for a monthly subscription between 15 and 20 Euro/month

\footnotetext{
${ }^{1}$ https://www.bluemovement.nl/abonnementen
} 
1 depending on the product features. The maximum duration of the subscription is however limited to 6

2 years. After this period the WM is refurbished or recycled.

3 Currently such PSS schemes are not common practice and in the baseline scenario no refurbishment or

4 component reuse is assumed. In the sensitivity analysis, however, a PSS scenario is investigated

\section{$5 \quad$ Collection at end-of-use}

6 Waste of electrical and electronic equipment (e-waste) is a fast-growing waste stream with complex 7 characteristics. Rapid technology innovation and shortening product lifespans are among the factors

8 contributing to the growing amount of e-waste [61]. Globally, only $8.9 \mathrm{Mt}$ of e-waste are documented to

9 be collected and recycled, which corresponds to $19.9 \%$ of all the e-waste generated [62]. Large household

10 equipment, such as washing machines and refrigerators, represent around $45 \%$ of the generated and

11 collected e-waste [61]-[63]. Although the annual collection rate is increasing in Europe, efforts are still

12 required to meet the target of $65 \%$ by the end of 2019 [64]

13 For the baseline, a global average collection rate for WM of $19.9 \%$ is taken into account and the effect of 14 increased collection rates is included in the scenario analysis.

\section{Recycling efficiencies}

16 Most collected e-waste is treated in a dedicated recycling plant. The treatment starts with size reduction 17 to liberate the different material fractions. The ferrous fraction is removed magnetically. Other non18 ferrous metals, such as aluminium and copper, are removed with an eddy current seperator. Current precious metal content in large household appliances, such as WMs, is too small to justify dedicated shredding and separation. Extensive research was performed by Huisman et al. to establish the recovery of the main fractions (ferrous, aluminium and copper). For metal dominated electronics, the efficiency of the ferrous recovery was found to be as high as $95 \%$. The recovery of the non-ferrous was lower with an estimated efficiency of $82,6 \%$ for aluminium and $78,2 \%$ for copper.

Ruan et al. found that a traditional eddy current separator offered low separation efficiency of non-ferrous metallic particles from crushed e-waste [65]. Marra et al. found that only $40 \%$ of the aluminium present in the input e-waste could be traced to the aluminium output fractions, while more than $70 \%$ of the total copper was sent to the corresponding output fraction [66]. The different degree of separation observed is closely related to the form in which each metal is present in the input material. Aluminium is more often found as an alloy or encapsulated in multi-material agglomerates [66], [67].

In 2015, a material flow analysis was conducted by sampling experiments at an e-waste treatment plant in Belgium [68]. The losses were estimated by sampling the resulting output fractions after each separation step. Based on the concentration and the output fraction mass, the separation efficiency of the magnet for ferrous metal recovery and the eddy current for non-ferrous metal were estimated at $92.95 \%$ and 


\subsection{Product Circularity Indicator (PCI) -baseline results}

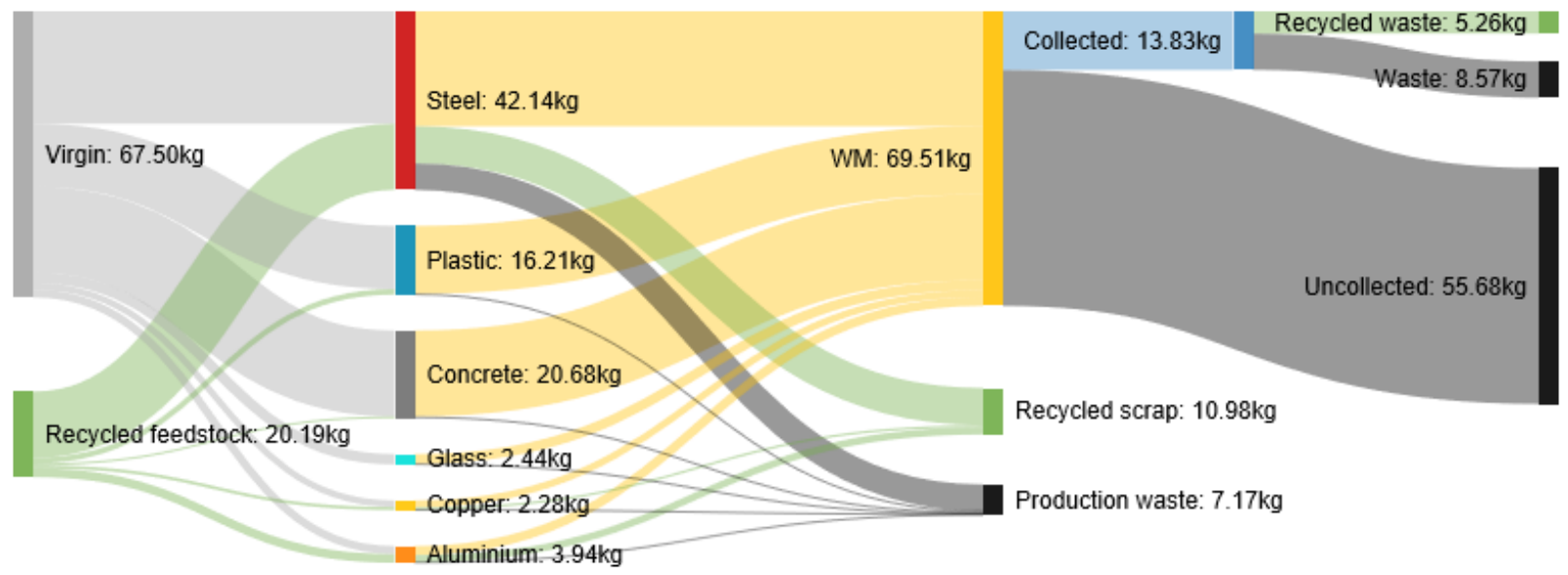

Figure 5: Overview of material flow through the value chain of a washing machine

The collected data, as described in the previous section, are summarized in Figure 5 . In total $87.72 \mathrm{~kg}$ of input is required for the manufacture of the WM even though the product only weighs $69.51 \mathrm{~kg}$. Recycled feedstock represents $23 \%$ of the required input material. The metals account for most of the recycled feedstock used, but they also are responsible for most of the material losses during production. Due to the recyclable properties of the metals, $61 \%$ of these material losses during manufacturing are kept in the material cycle as recycled scrap. Other materials, such as plastic and concrete, have a limited recycled content, but generate much less production waste. Obviously, the low collection rate at end-of-life is a major loss for WMs (and other electronic products) in terms of material efficiency. In addition, only the metals are successfully recovered from the collected WM. Consequently, on average only $38 \%$ of the collected WM material is recovered. The overall calculated $\mathrm{PCl}$ of the WM is 0.149 . Considering the $\mathrm{PCl}$ can vary between 0 and 1 , this is a rather low score. Potential improvement measures to increase the $\mathrm{PCl}$ are discussed in the next section.

\subsection{Scenario analysis for improvement strategies based on CE thinking} Selecting more recyclable materials is sometimes assumed to increase the overall circularity performance of a product. The recyclable "material selection" scenario investigates the effect of replacing concrete with steel (cast iron) for the counterweight component. Such WMs have already been introduced on the market but are not very common due to the difference in material price between concrete and steel.

Increasing material recycling is an often used strategy to increase the circularity of product systems. As shown in Figure 5, the overall low collection rate for electronic waste results in a significant amount of unrecoverable waste. The "collection rate" scenario estimates the effect of current ambitioned European collection rates. The "material recycling" scenario investigates the effect of increasing the recycling of plastic, glass and concrete material. However, there are technical limitations to the potential improvement of plastic recycling. First, some plastic types, such as fibre reinforced plastic (FRP), are considered unrecyclable. While others, such as Polymethyl methacrylate (PMMA), are present in such low concentration that separating them during waste treatment at end-of-use is not practically feasible. Both recycled content of the input material $\left(F_{r}=0.5\right)$ and recovery at end-of-use $\left(E_{m s}=0.65\right.$ and $E_{r f p}=0.9$ ) 
1 are considered. Finally, the "enhanced recycling" scenario combines the increased collection rate and 2 material recycling.

3 Product System Services (PSS) are often referred to as a promising CE strategy. Such a strategy can only be $4 \quad$ viable if the product is well-managed at the end-of-use and increased collection rates are assumed to be 5 a consequence of the business model choice. The supplier does not sell the WM but only the service of 6 using the WM which means there is no transfer of ownership. Next to the improved collection and waste 7 management, such a business model could also increase the useful lifetime of the WM parts by introducing 8 regular maintenance and refurbishment. Based on manufacturer's expectation, the "PSS" scenario 9 assumes that the WM is refurbished every 6 years and that on average parts are used 3 times. The utility factor is therefore equal to 0.6 assuming the wash frequency is unchanged. To incorporate the number of uses $(N=3)$ in our steady-state model, the collection for parts $\left(C_{u}\right)$ and the fraction of reused parts in each WM $\left(F_{u}\right)$ are calculated as follows:

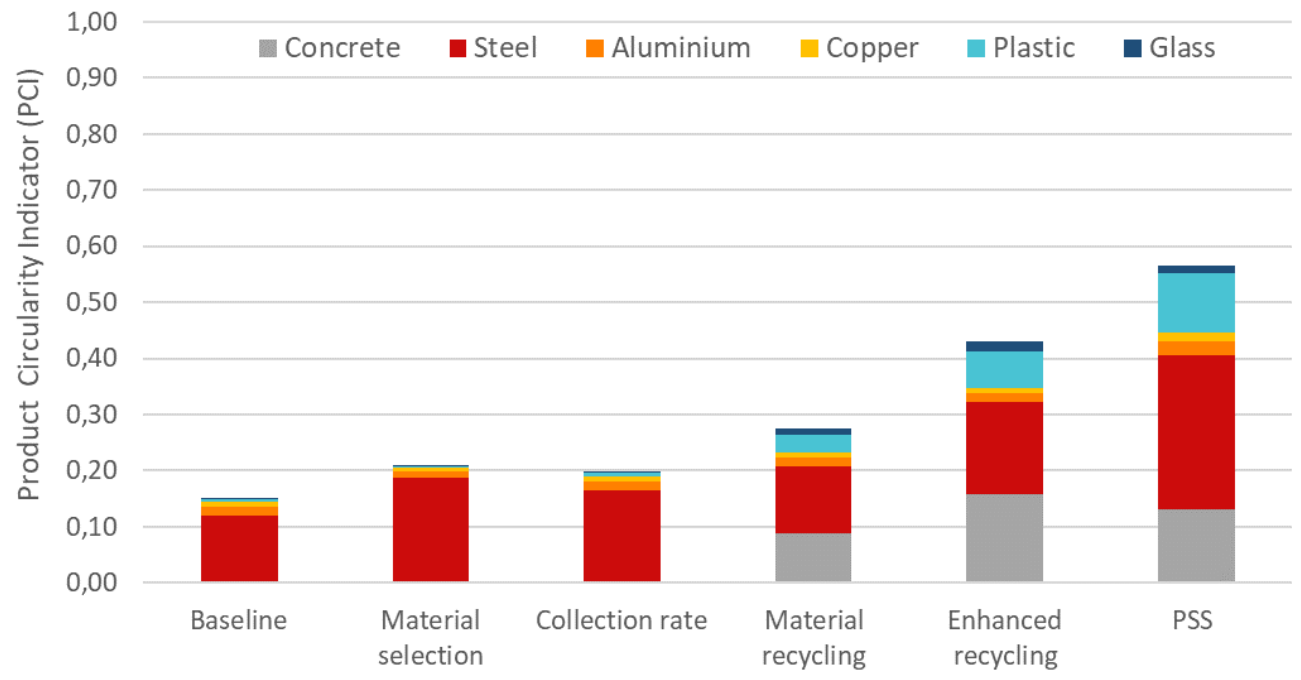

Figure 6: $\mathrm{PCl}$ results for scenario analysis for improvement strategies based on $\mathrm{CE}$ thinking

The $\mathrm{PCI}$ results, shown in Figure 6, confirm that the improvement strategies based on CE thinking improve the circularity of the product. Substituting concrete for a more recyclable material can have a positive effect on the circularity performance of the product $(\mathrm{PCl}=0.208)$. The circularity improvement with increased collection rate is limited due to the overall low recovery for the WM. While increasing the material recycling of currently unrecovered materials, such as plastic, concrete and glass, increases the $\mathrm{PCl}$ up to 0.276 , the results of combined efforts are significantly improved $(\mathrm{PCl}=0.430)$. Although the current envisioned PSS by the WM manufacturer results in a clear $\mathrm{PCl}$ increase $(\mathrm{PCl}=0.566)$, it can achieve a higher circularity score by combining it with improved recycling or increasing the durability of the WM and its' parts $(X \geq 1)$. 


\subsection{Potential trade-offs with environmental performance}

2 In this section, the potential trade-off between increasing circularity and minimising the environmental 3 burden of the WM is investigated. The environmental performance of the different scenario's from 4 previous section is quantified in a comparative, attributional life cycle assessment (LCA) approach, using 5 the Ecoinvent 3.3 database and the ReCiPe $(\mathrm{H} / \mathrm{A})$ endpoint method with European dataset. Although the 6 ReCiPe method has been updated in 2016, the normalisation and weighting, which allows to aggregate 7 the results in a single score, has not yet been finalized. System expansion (ISO 14040:2006) is used to 8 assure comparability of different scenarios, hence all providing the same 'basket of products'. The 9 functional unit used for this analysis is defined as the use of one WM for clothes washing, with a lifetime expectancy of 2500 wash cycles.

The environmental impact assessment includes the following stages: (1) material production, (2) product manufacturing, (3) waste management and (4) recycling. The material production is related to the amount of virgin material that needs to be extracted and refined. The product manufacturing includes both the production of feedstock and the final component fabrication. The material losses during manufacturing are taken into account and have been quantified in previous section. The assembly phase is not significantly altered in the different scenario's because it will always take place with both new and reused components. For the use phase, an energy consumption in real-life conditions of $0.672 \mathrm{kWh} /$ wash cycle is assumed [69]. The waste management handles waste from uncollected products, production waste and unrecycled rest fraction after material separation. The uncollected products at end-of-life are assumed to landfilled while the production and unrecycled waste are assumed to be incinerated. The collected products are assumed the be shredded followed by magnetic and eddy current separation. The metal fractions are further refined to produced recycled material that can serve for new feedstock production.

Figure 7 (a) shows the LCA results for the baseline and different improvement strategies based on CEthinking. Due to the high energy consumption during each wash cycle, the use phase is identified as the life stage with the highest environmental burden. This identifies the first potential trade-off when dealing with energy-using product because the circularity measure does not take into account the burden of energy requirement during the use phase. A second trade-off identified, is the fact that selecting more recyclable material, such as steel vs. concrete, can potentially increase the overall burden.

Figure 7 (b) shows the LCA results without the dominating use phase and includes the environmental impact of the virgin material extraction for each material. The PWBs have a significant contribution in terms of environmental burden which is completely overlooked when focussing on the circularity of material streams. Nevertheless, the LCA results confirm the envisioned PSS strategy will both increase the circularity and reduce the environmental burden of the product system if the WM can indeed be successfully refurbished every 6 year and the majority of the components can be reused 3 times. 


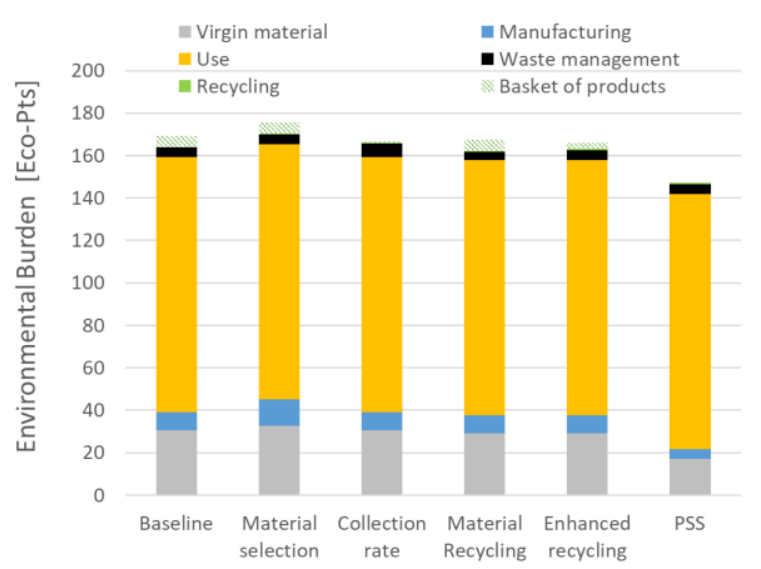

(a)

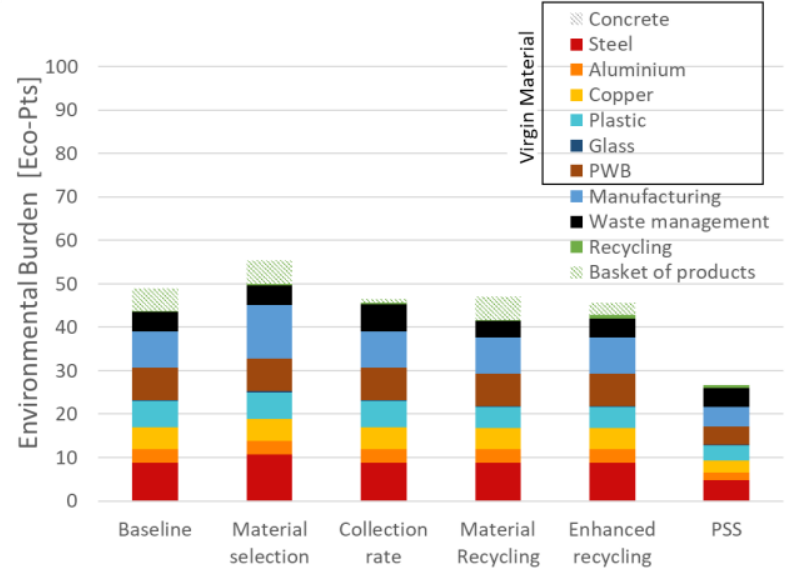

(b)

Figure 7: LCA results for scenario analysis for improvement strategies based on CE thinking (a) including the use phase (b) excluding the use and indicating the contribution of each raw material extraction 
2 This paper researches the possibility to measure the performance of more circular complex product supply chains. Although there are already a number of circularity indicators proposed in literature, none was found to properly describe the product system. The Material Circularity Indicator $(\mathrm{MCl})$, proposed by the EMF and GD, is one of the most cited metrics at product-level. However, the indicator is unable to account for the 'tightness' of the material cycles and ignores the relationship with other product system for example for the use or supply of recycled material. In addition, the indicator does not take into account material losses during manufacturing. Therefore, a new Product Circularity Indicator ( $\mathrm{PCI}$ ) is developed in this paper and applied in a case study.

Although the indicator is defined at product-level, a part of the dataset is collected at larger scale. For example the material production is not specific to WMs, so the average manufacturing data for semifabricated products are assumed to be representative for our case study. Unless a specific take-back scheme is put in place, the product manufacturers also have limited influence on the collected rate and end-of-life management of discarded products, and thus the use of collection data at country or regional level are relevant.

The case study results show that the proposed $\mathrm{PCl}$ is a useful indicator to investigate the effectiveness of different CE strategies such as reuse of component in a Product Service Systems (PSS). The PCl can also quantify the benefits in terms of material efficiency of increased recycling, although to be effective the product should both use and supply recycled material. Finally, the $\mathrm{PCI}$ results demonstrate the importance of combining complementary CE strategies at different stages of the product cycle.

Nevertheless, there are some limitation to the proposed indicators that need to be taken into account and could form the subject of future work. Currently, the different quality of recycled materials is not taken into account due to the lack of an appropriate quality factor that measures quality degradation for different material types. In absence of such a measure, the material value or price could be used a proxy. In addition, such a weighting based on value could also be applied to the different materials included in the product. In that case, more importance would be given to precious metals often present at low concentration. The mining or material extraction stage is currently not included in the product system. Although manufacturer and designer can choose a specific material, the origin of it is very difficult to trace back. However, as ore concentration are expected to decline and new deposits become more scarce, it could be useful to include this stage in future. Finally, it is important to stress the indicator only measures the circularity of the flows. Other effects on the environment, typically assessed with a Life Cycle Assessment, LCA, are not covered. Potential trade-offs between increasing circularity and minimizing environmental burden should not be ignored.

\section{Acknowledgements}

35 The authors acknowledge support of the research fund of KU Leuven through project GOA/15/01236 SUMMA. 


\section{References}

[1] European Commission, "Decision No 1386/2013/EU of the European Parliament and of the Council of 20 November 2013 on a General Union Environment Action Programme to 2020 'Living well, within the limits of our planet' Text with EEA relevance," 12-2013. [Online]. Available: http://ec.europa.eu/environment/action-programme/. [Accessed: 08-Jun-2018].

[2] K. Raworth, Doughnut economics: seven ways to think like a 21st century economist. White River Junction, Vermont: Chelsea Green Publishing, 2017.

[3] United Nations, "Sustainable Development Goals," Sep-2015. [Online]. Available: http://www.un.org/sustainabledevelopment/sustainable-development-goals.

[4] J. Rockström, "Planetary Boundaries," New Perspect. Q., vol. 27, no. 1, pp. 72-74, Jan. 2010.

[5] D. Giurco and C. Cooper, "Mining and sustainability: asking the right questions," Miner. Eng., vol. 29, pp. 3-12, Mar. 2012.

[6] G. M. Mudd, "The Environmental sustainability of mining in Australia: key mega-trends and looming constraints," Resour. Policy, vol. 35, no. 2, pp. 98-115, Jun. 2010.

[7] J. E. Tilton, On borrowed time? Assessing the threat of mineral depletion. Washington, DC: Resources for the Future, 2003.

[8] J. Dewulf et al., "Rethinking the Area of Protection 'Natural Resources' in Life Cycle Assessment," Environ. Sci. Technol., vol. 49, no. 9, pp. 5310-5317, May 2015.

[9] T. Sonderegger et al., "Towards harmonizing natural resources as an area of protection in life cycle impact assessment," Int. J. Life Cycle Assess., vol. 22, no. 12, pp. 1912-1927, Dec. 2017.

[10] W. Haas, F. Krausmann, D. Wiedenhofer, and M. Heinz, "How Circular is the Global Economy?: An Assessment of Material Flows, Waste Production, and Recycling in the European Union and the World in 2005: How Circular is the Global Economy?," J. Ind. Ecol., vol. 19, no. 5, pp. 765-777, Oct. 2015.

[11] European Commission, "Closing the loop - An EU action plan for the Circular Economy." 12-Feb-2015.

[12] Global Footprint Network, "World Footprint," 2018. [Online]. Available: https://www.footprintnetwork.org/our-work/ecological-footprint/\#worldfootprint. [Accessed: 08Jun-2018].

[13] UNEP, Decoupling natural resource use and environmental impacts from economic growth. Kenya, UNEP, 2011.

[14] L. Beaulieu, G. van Durme, M.-L. Arpin, and CIRAIG, Circular economy: a critical literature review of concepts. 2016.

[15] F. Blomsma and G. Brennan, "The Emergence of Circular Economy: A New Framing Around Prolonging Resource Productivity: The Emergence of Circular Economy," J. Ind. Ecol., vol. 21, no. 3, pp. 603-614, Jun. 2017.

[16] P. Ghisellini, C. Cialani, and S. Ulgiati, "A review on circular economy: the expected transition to a balanced interplay of environmental and economic systems," J. Clean. Prod., vol. 114, pp. 11-32, Feb. 2016.

[17] M. Linder, S. Sarasini, and P. van Loon, "A Metric for Quantifying Product-Level Circularity: ProductLevel Circularity Metric," J. Ind. Ecol., vol. 21, no. 3, pp. 545-558, Jun. 2017.

[18] K. Webster, "What Might We Say about a Circular Economy? Some Temptations to Avoid if Possible," World Futur., vol. 69, no. 7-8, pp. 542-554, Nov. 2013.

[19] Ellen MacArthur Foundation, "Towards a circular economy: Business rationale for an accelerated transition," Dec. 2015.

[20] J. M. Cullen, "Circular Economy: Theoretical Benchmark or Perpetual Motion Machine?: CE: Theoretical Benchmark or Perpetual Motion Machine?," J. Ind. Ecol., vol. 21, no. 3, pp. 483-486, Jun. 2017. 
[21] F. De Carlo, O. Borgia, and M. Tucci, "Accelerated degradation tests for reliability estimation of a new product: A case study for washing machines," Proc. Inst. Mech. Eng. Part O J. Risk Reliab., vol. 228, no. 2, pp. 127-138, Apr. 2014.

[22] Ellen MacArthur Foundation and Granta Design, "Circularity indicators - An approach to measuring circularity - methodology," May 2015.

[23] J. A. Garza-Reyes, A. Salomé Valls, S. Peter Nadeem, A. Anosike, and V. Kumar, "A circularity measurement toolkit for manufacturing SMEs," Int. J. Prod. Res., pp. 1-25, Dec. 2018.

[24] G. Lonca, R. Muggéo, H. Imbeault-Tétreault, S. Bernard, and M. Margni, "Does material circularity rhyme with environmental efficiency? Case studies on used tires," J. Clean. Prod., vol. 183, pp. 424435, May 2018.

[25] G. Moraga et al., "Circular economy indicators: What do they measure?," Resour. Conserv. Recycl., vol. 146, pp. 452-461, Jul. 2019.

[26] M. Niero and M. Z. Hauschild, "Closing the Loop for Packaging: Finding a Framework to Operationalize Circular Economy Strategies," Procedia CIRP, vol. 61, pp. 685-690, 2017.

[27] A. Selvefors, O. Rexfelt, S. Renström, and H. Strömberg, "Use to use - A user perspective on product circularity," J. Clean. Prod., vol. 223, pp. 1014-1028, Jun. 2019.

[28] Ž. Zore, L. Čuček, and Z. Kravanja, "Synthesis of sustainable production systems using an upgraded concept of sustainability profit and circularity," J. Clean. Prod., vol. 201, pp. 1138-1154, Nov. 2018.

[29] M. Saidani, B. Yannou, Y. Leroy, F. Cluzel, and A. Kendall, "A taxonomy of circular economy indicators," J. Clean. Prod., vol. 207, pp. 542-559, Jan. 2019.

[30] Y. Geng, J. Fu, J. Sarkis, and B. Xue, "Towards a national circular economy indicator system in China: an evaluation and critical analysis," J. Clean. Prod., vol. 23, no. 1, pp. 216-224, Mar. 2012.

[31] S. Pauliuk, G. Majeau-Bettez, and D. B. Müller, "A General System Structure and Accounting Framework for Socioeconomic Metabolism: General System Structure for Society's Metabolism," J. Ind. Ecol., vol. 19, no. 5, pp. 728-741, Oct. 2015.

[32] H. Schandl, D. B. Müller, and Y. Moriguchi, "Socioeconomic Metabolism Takes the Stage in the International Environmental Policy Debate: A Special Issue to Review Research Progress and Policy Impacts: SEM: A Special Issue to Review Research Progress," J. Ind. Ecol., vol. 19, no. 5, pp. 689-694, Oct. 2015.

[33] F. Di Maio and P. C. Rem, "A Robust Indicator for Promoting Circular Economy through Recycling," J. Environ. Prot., vol. 06, no. 10, pp. 1095-1104, 2015.

[34] J. Y. Park and M. R. Chertow, "Establishing and testing the 'reuse potential' indicator for managing wastes as resources," J. Environ. Manage., vol. 137, pp. 45-53, May 2014.

[35] V. Elia, M. G. Gnoni, and F. Tornese, "Measuring circular economy strategies through index methods: A critical analysis," J. Clean. Prod., vol. 142, pp. 2741-2751, Jan. 2017.

[36] M. Niero and P. P. Kalbar, "Coupling material circularity indicators and life cycle based indicators: A proposal to advance the assessment of circular economy strategies at the product level," Resour. Conserv. Recycl., vol. 140, pp. 305-312, Jan. 2019.

[37] S. Jayatilleka, "Performance Verification Throughout the Product Life Cycle Using Accelerated Life Testing," in 2018 Annual Reliability and Maintainability Symposium (RAMS), Reno, NV, 2018, pp. 16.

[38] Z. Chen, T. Zhao, S. Luo, and Y. Sun, "Warranty Cost Modeling and Warranty Length Optimization Under Two Types of Failure and Combination Free Replacement and Pro-Rata Warranty," IEEE Access, vol. 5, pp. 11528-11539, 2017.

[39] D. L. Schrijvers, P. Loubet, and G. Sonnemann, "Developing a systematic framework for consistent allocation in LCA," Int. J. Life Cycle Assess., vol. 21, no. 7, pp. 976-993, Jul. 2016. 
[40] C. Koffler and J. Florin, "Tackling the Downcycling Issue-A Revised Approach to Value-Corrected Substitution in Life Cycle Assessment of Aluminum (VCS 2.0)," Sustainability, vol. 5, no. 11, pp. 45464560, Oct. 2013.

[41] R. Stamminger, P. Tecchio, F. Ardente, F. Mathieux, and P. Niestrath, "Towards a durability test for washing-machines," Resour. Conserv. Recycl., vol. 131, pp. 206-215, Apr. 2018.

[42] P. Tecchio, R. Stamminger, F. Ardente, P. Niestrath, and F. Mathieux, Study for the development of an endurance testing method for washing machines. Luxembourg: Joint Research Centre, 2017.

[43] E. Bracquené et al., "Repairability criteria for energy related products - Study in the BeNeLux context to evaluate the options to extend the product life time - Final Report." 14-Jun-2018.

[44] RREUSE, "Investigation into the repairability of Domestic Washing Machines, Dishwashers and Fridges," Dec. 2013.

[45] WRAP, "Specifying durability and repair in washing machines," Jun. 2011.

[46] A. M. Bundgaard, A. Remmen, and K. Overgaard Zacho, Ecodesign Directive version 2.0 - From Energy Efficiency to Resource Efficiency Environmental project No. 1635, 2015. Miljøstyrelsen, 2015.

[47] T. Devoldere, B. Willems, J. R. Duflou, and W. Dewulf, "The eco-efficiency of reuse centres critically explored - the washing machine case," Int J Sustain. Manuf., vol. 1, no. 3, pp. 265-285, 2009.

[48] I. Rüdenauer, C. Gensch, and D. Quack, "Eco-Efficiency Analysis of Washing machines - Life Cycle Assessment and determination of optimal life span," Öko-Institut, Freiburg, Nov. 2006.

[49] M. F. Ashby, "Case studies," in Materials and the Environment, Elsevier, 2013, pp. 193-225.

[50] M. Oguchi, S. Murakami, H. Sakanakura, A. Kida, and T. Kameya, "A preliminary categorization of end-of-life electrical and electronic equipment as secondary metal resources," Waste Manag., vol. 31, no. 9-10, pp. 2150-2160, Sep. 2011.

[51] D. Kawecki, P. R. W. Scheeder, and B. Nowack, "Probabilistic Material Flow Analysis of Seven Commodity Plastics in Europe," Environ. Sci. Technol., vol. 52, no. 17, pp. 9874-9888, Sep. 2018.

[52] M. Bertram et al., "A regionally-linked, dynamic material flow modelling tool for rolled, extruded and cast aluminium products," Resour. Conserv. Recycl., vol. 125, pp. 48-69, Oct. 2017.

[53] S. Glöser, M. Soulier, and L. A. Tercero Espinoza, "Dynamic Analysis of Global Copper Flows. Global Stocks, Postconsumer Material Flows, Recycling Indicators, and Uncertainty Evaluation," Environ. Sci. Technol., vol. 47, no. 12, pp. 6564-6572, Jun. 2013.

[54] B. Scalet, M. Garcia Munoz, A. Sissa, S. Roudier, and L. Delgado Sancho, "Best Available Techniques (BAT) Reference Document for the Manufacture of Glass," Joint Research Centre, JRC 78091, 2013.

[55] J. M. Cullen, J. M. Allwood, and M. D. Bambach, "Mapping the Global Flow of Steel: From Steelmaking to End-Use Goods," Environ. Sci. Technol., vol. 46, no. 24, pp. 13048-13055, Dec. 2012.

[56] R. Hischier, "Life Cycle Inventories of Packaging and Graphical papers - Part II Plastics," Empa, Dübendorf, 11, 2007.

[57] World Aluminium, "Global aluminium cycle 2017," 2018. [Online]. Available: http://www.worldaluminium.org/statistics/massflow/.

[58] O. Borgia, F. D. Carlo, N. Fanciullacci, and M. Tucci, "Accelerated life tests for new product qualification: a case study in the household appliance," IFAC Proc. Vol., vol. 46, no. 7, pp. 269-274, May 2013.

[59] A. Kruschwitz, A. Karle, A. Schmitz, and R. Stamminger, "Consumer laundry practices in Germany: Consumer laundry practices in Germany," Int. J. Consum. Stud., vol. 38, no. 3, pp. 265-277, May 2014.

[60] A. Boyano et al., Ecodesign and Energy Label for Household Dishwashers - Preparatory study Final Report. 2017.

[61] C. P. Balde, The global e-waste monitor 2014: quantities, flows and resources. United Nations University, 2015. 
[62] C. P. Balde et al., The Global E-waste Monitor - 2017 Baldé, C.P., Forti V., Gray, V., Kuehr, R., Stegmann, P. 2017.

[63] European Union, "eurostat - European statistics - Waste electrical and electronic equipment (WEEE) by waste management operations [env_waselee]," 2019. [Online]. Available: http://ec.europa.eu/eurostat/data/database.

[64] European Commission, "Directive 2012/19/EU of The European Parliament and of the Council of 4 July 2012 on waste electrical and electronic equipment (WEEE) (recast)'." 2012.

[65] J. Ruan and Z. Xu, "Approaches To Improve Separation Efficiency of Eddy Current Separation for Recovering Aluminum from Waste Toner Cartridges," Environ. Sci. Technol., vol. 46, no. 11, pp. 62146221, Jun. 2012.

[66] A. Marra, A. Cesaro, and V. Belgiorno, "Separation efficiency of valuable and critical metals in WEEE mechanical treatments," J. Clean. Prod., vol. 186, pp. 490-498, Jun. 2018.

[67] Z. H. I. Sun, Y. Xiao, J. Sietsma, H. Agterhuis, G. Visser, and Y. Yang, "Characterisation of metals in the electronic waste of complex mixtures of end-of-life ICT products for development of cleaner recovery technology," Waste Manag., vol. 35, pp. 227-235, Jan. 2015.

[68] J. R. Duflou, J. R. Peeters, D. Altamirano, E. Bracquene, and W. Dewulf, "Demanufacturing photovoltaic panels: Comparison of end-of-life treatment strategies for improved resource recovery," CIRP Ann., vol. 67, no. 1, pp. 29-32, 2018.

[69] P. Tecchio, F. Ardente, and F. Mathieux, "Analysis of durability, reusability and reparability Application to washing machines and dishwashers," EUR 28042 EN, 2016. 